\title{
ESTUDIO DE LOS PARÁMETROS DE LA CABEZA DEL NERVIO ÓPTICO EN SUJETOS NORMALES, HIPERTENSOS OCULARES Y GLAUCOMATOSOS OBTENIDOS MEDIANTE LÁSER CONFOCAL DE BARRIDO (HEIDELBERG RETINA TOMOGRAPH II)
}

\section{OPTIC NERVE HEAD PARAMETERS AS MEASURED BY CONFOCAL SCANNING LASER (HEIDELBERG RETINA TOMOGRAPH II) IN NORMAL, OCULAR HYPERTENSIVE AND GLAUCOMATOUS SUBJECTS}

\author{
LARROSA JM ${ }^{1}$, POLO V ${ }^{1}$, PÉREZ-ÍNIIGO A ${ }^{2}$, FERRERAS A ${ }^{2}$, GARCÍA-FEIJOÓ J ${ }^{3}$, ANTÓN A ${ }^{4}$, \\ HONRUBIA FM ${ }^{5}$
}

\section{RESUMEN}

Objetivo: Comparar los parámetros del nervio óptico obtenidos mediante láser confocal en sujetos normales, hipertensos oculares y glaucomatosos y en los subgrupos de hipertensos clasificados según el aspecto papilar y la perimetría automatizada de longitud de onda corta (PALOC).

Métodos: Se incluyeron 101 ojos de 101 sujetos normales, 247 ojos de 247 hipertensos oculares y 102 ojos de 102 glaucomatosos. Los sujetos fueron clasificados según la presión intraocular y resultados de la perimetría automatizada (PA). Se clasificó a los hipertensos en hipertensos oculares y glaucomas preperimétricos, y en hipertensos oculares con PALOC normal e hipertensos con PALOC alterada. Se realizó evaluación papilar, PA y PALOC y

\section{ABSTRACT}

Purpose: To compare the optic nerve parameters measured by confocal scanning laser in normal, ocular hypertensive and glaucomatous eyes; and in groups of ocular hypertensive eyes, classification of these according to the optic nerve appearance and to short-wavelength automated perimetry (SWAP) results.

Methods: 101 eyes of 101 normal subjects, 247 eyes of 247 ocular hypertensive subjects and 102 eyes of 102 glaucomatous subjects were studied. Subjects were classified based on intraocular pressure and standard automated perimetry (AP) performance. Hypertensive subjects were classified into ocular hypertensive and preperimetric glaucoma, and into ocular hypertensive with normal SWAP and hypertensive with pathological SWAP findings.

\footnotetext{
Recibido: 11/5/05. Aceptado: 9/6/08. Glaucoma.

Correspondencia:

M. ${ }^{a}$ Aránzazu Pérez Íñigo

Servicio de Oftalmología

Hospital Universitario Miguel Servet

Isabel la Católica, 1-3

50009 Zaragoza

España

E-mail: aperezinigo@hotmail.com
}

1 Doctor en Medicina. Profesor titular de Oftalmología. Hospital Universitario Miguel Servet. Zaragoza. España.

2 Doctor en Medicina. Hospital Universitario Miguel Servet. Zaragoza. España.

3 Doctor en Medicina. Profesor titular de Oftalmología. Hospital Clínico San Carlos. Madrid. España.

4 Doctor en Medicina. Profesor asociado de Oftalmología. Hospital de la Esperanza. Barcelona. España.

5 Doctor en Medicina. Catedrático de Oftalmología. Hospital Universitario Miguel Servet. Zaragoza. España.

Este trabajo ha sido parcialmente financiado por la Red Nacional de Investigación Cooperativa: Prevención de la Ceguera. Subproyecto de 
un estudio mediante láser confocal Heidelberg Retina Tomograph II obteniendo los valores de las variables topográficas del nervio, que fueron comparados entre los grupos, así como entre cada subgrupo de hipertensos y el grupo de sujetos normales y glaucomatosos.

Resultados: Se observaron diferencias significativas $(\mathrm{p}<0,05)$ entre normales y glaucomatosos en todos los parámetros excepto área papilar $(2,23 / 2,1)$, variación de altura de contorno $(0,41 / 0,39)$ y variabilidad media $(0,05 / 0,04)$. Los hipertensos mostraron solapamiento de resultados respecto a controles y glaucomatosos, no hubo diferencias entre hipertensos y controles en grosor de capa de fibras $(0,24 / 0,24)$ o entre hipertensos y glaucomas en profundidad media de excavación $(0,28 / 0,3)$. Sin embargo, la división de hipertensos según el aspecto papilar o sus resultados en la PALOC mejoró la capacidad de discriminación entre grupos.

Conclusiones: El HRT proporciona moderada capacidad de discriminación entre sujetos normales, hipertensos oculares y glaucomatosos. Otras herramientas (como la PALOC) mejoran la capacidad discriminatoria del HRT.

Palabras clave: Glaucoma, diagnóstico, HRT, nervio óptico.
Every patient underwent evaluation of the optic nerve, AP, SWAP and confocal scanning laser ophthalmoscopy (HRT II), resulting in the acquisition of topographic parameters of the optic nerve, which were then compared between the different groups.

Results: Significant differences $(\mathrm{p}<0.05)$ were found between normal subjects and glaucomatous eyes in all optic nerve parameters except disc area $(2.23 / 2.1)$, height variation contour $(0.41 / 0.39)$ and average variability $(0.05 / 0.04)$. Ocular hypertensive eyes showed an overlap when compared with normal and glaucoma groups. No differences were found between normal subjects and those with ocular hypertension in mean retinal nerve fiber layer thickness $(0.24 / 0.24)$ or between those with ocular hypertension and glaucoma in mean cup depth $(0.28 / 0.3)$. Nevertheless, when those with ocular hypertension were segregated based on clinical evaluation of optic nerve or SWAP performance, the ability to define the presence of structural glaucoma damage improved significantly.

Conclusions: HRT enables moderate discrimination between normal, ocular hypertensive and glaucoma subjects. The use of diagnostic tests to detect early glaucomatous damage (such as short-wavelength automated perimetry) in ocular hypertensive eyes improves the ability of HRT to discriminate glaucoma (Arch Soc Esp Oftalmol 2008; 83: 407-416).

Key words: Glaucoma, diagnosis, HRT, optic nerve head.

\section{INTRODUCCIÓN}

El glaucoma crónico simple es una neuropatía óptica progresiva con una importante incidencia en nuestro medio (1-6).

La evaluación del nervio óptico ha constituido un pilar imprescindible en el diagnóstico (7-12). La apreciación de cualquier cambio sugestivo de glaucoma, está sujeta a la experiencia y subjetividad del observador. Esto ha condicionado que se hayan desarrollado técnicas de evaluación objetiva y mediciones cuantitativas de las estructuras del nervio óptico buscando una mayor precisión diagnóstica, mejor reproducibilidad y menor dependencia de la interpretación por parte del explorador.

El Tomógrafo Retiniano Heidelberg - HRT IIrepresenta un láser confocal de barrido de última generación que permite obtener imágenes morfo- métricas del nervio óptico realizando un análisis papilar objetivo y reproducible.

El objetivo principal del presente estudio es comparar los parámetros morfométricos papilares obtenidos mediante un láser confocal de barrido (HRT II) en una población de sujetos normales, hipertensos oculares y glaucomatosos.

El objetivo secundario es comparar los datos del HRT de la población normal y glaucomatosa respecto a pacientes hipertensos oculares con PALOC alterada o valoración clínica del nervio óptico anómala.

\section{MATERIAL Y MÉTODOS}

\section{Sujetos}

Se han incluido en el presente estudio un total de 450 ojos de 450 sujetos. Cada sujeto, cumplía una 
serie de criterios de inclusión, que fueron: edad comprendida entre 30 y 80 años, agudeza visual igual o superior a $8 / 10$ (escala de Snellen), defecto de refracción inferior a 5.00 dioptrías de equivalente esférico y 3.00 dioptrías de cilindro y medios ópticos transparentes. Se obtuvo consentimiento informado.

Se excluyeron los sujetos con antecedentes de patología, cirugía o traumatismo oculares, enfermedades sistémicas de repercusión oftálmica e imposibilidad de realizar o valorar cualquiera de las pruebas incluidas en el protocolo.

Los sujetos incluidos fueron clasificados en tres grupos de estudio: control, hipertensión ocular (HTO) y glaucoma.

- Grupo Control (n = 101 ojos). Debían cumplir los criterios de inclusión y un criterio adicional, ya que la presión intraocular debía ser $<$ o = a 21 $\mathrm{mm} \mathrm{Hg}$ en todas las determinaciones. La Perimetría Automatizada (PA) debía ser normal.

- Grupo de HTO ( $\mathrm{n}=247$ ojos) Presentaban el hallazgo, en al menos dos ocasiones, de cifras de presión intraocular mayores de $21 \mathrm{~mm} \mathrm{Hg}$, así como un estudio de PA absolutamente normal.

Subgrupos:

En base a la evaluación clínica del nervio óptico:

- Hipertensos oculares sin evidencias de sospecha de daño papilar (hipertensos oculares) $n=126$.

- Hipertensos oculares altamente sospechosos de daño glaucomatoso papilar (glaucomas preperimétricos) $n=121$.

En base a los resultados del estudio de PALOC:

Hipertensos oculares sin pérdidas funcionales en la PALOC. $n=123$

Hipertensos oculares con alteración en la PALOC. $\mathrm{n}=46$

- Grupo de Glaucoma ( $\mathrm{n}=102$ ojos) Presentaban cifras tensionales elevadas (PIO > $21 \mathrm{~mm} \mathrm{Hg}$ ) y defectos campimétricos en la PA. Los sujetos con glaucoma no fueron seleccionados en base a la evaluación clínica papilar sino basándonos en la valoración de la perimetría automatizada convencional (prueba diagnóstica de referencia).

\section{Procedimiento exploratorio}

Incluía:

- Perimetría Automatizada Convencional, realizada con el Analizador de Campo «HUMPHREY 630» (30-II, STACPAC 2). Los criterios para definir una perimetría como alterada fueron: presencia de grupos de puntos con descensos significativos de sensibilidad (al menos tres puntos contiguos en el mismo hemicampo con nivel de significación $\mathrm{p}<0,05$ o un grupo de al menos dos puntos contiguos en el mismo hemicampo con nivel de significación $\mathrm{p}<0,01)$ y/o una Desviación Estándar Patrón Corregida (CPSD) estadísticamente significativas, a niveles de $\mathrm{p}<0,02$ y/o presencia de un Glaucoma Hemifield Test (GHT) fuera de los límites de la normalidad.

- Perimetría Automatizada de Longitud de Onda Corta (PALOC), realizada con el Analizador de Campo «HUMPHREY 630 modificado» (30-II, STACPAC 2). El criterio utilizado para definir la perimetría como anormal fue la presencia de un grupo de al menos cuatro puntos con un descenso de sensibilidad mayor de $5 \mathrm{~dB}(\mathrm{P}<0,05)$ o un grupo de al menos tres puntos con un descenso de sensibilidad mayor de $10 \mathrm{~dB}(\mathrm{p}<0,001)$ (estos puntos no se debían encontrar en la corona periférica del campo visual, ni en los vértices de la mancha ciega).

- Evaluación clínica del nervio óptico, empleando estereofotografías papilares. Se establecieron los siguientes criterios para definir un nervio óptico como altamente sospechoso de daño glaucomatoso: existencia de un adelgazamiento del anillo neurorretiniano focal o difuso y/o presencia de hemorragia papilar y/o existencia de muescas papilares y/o evidencia de una asimetría papilar en el cociente excavación/disco (E/D) vertical de al menos 2/10 entre ambos ojos.

- Láser Confocal de Barrido. Heidelberg Retina Tomograph — HRT II-, empleando un láser confocal de barrido (Heidelberg Retina Tomograph HRT version II) que permite obtener una imagen topográfica de la papila mediante una serie de imágenes de cortes ópticos de diferente profundidad. A partir de la imagen tridimensional reconstruida, el explorador establece y delimita el contorno del disco óptico. Esta delimitación la realizó un especialista en glaucoma y con experiencia en planimetría papilar (JL). La delimitación se llevó a cabo sin información del grupo de inclusión del sujeto ni de los resultados de la exploración. Tras la delimitación del contorno papilar el programa establece un plano de referencia de manera automática.

El HRT II incorpora un programa de análisis (Heidelberg Eye Explorer version 1.3.0.0) que calcula múltiples parámetros estructurales del nervio óptico: 
- Área de disco, Área de excavación, Área de anillo, Cociente área de excavación/disco, Cociente área de anillo/disco, Volumen de excavación, Volumen de anillo, Profundidad media de la excavación, Profundidad máxima de la excavación, Variación de la altura del contorno, Índice de morfología de la excavación («cup shape measure»-CSM-), Espesor de la capa de fibras nerviosas de la retina, Área de sección de la capa de fibras nerviosas de la retina, Cociente excavación/papila horizontal, Cociente excavación/papila vertical, Máxima elevación del contorno, Máxima depresión del contorno, Línea de modulación de contorno Temporal Superior, Línea de modulación de contorno Temporal Inferior, Variabilidad media, Altura de referencia.

Adicionalmente, el programa de análisis incorpora las siguientes funciones discriminantes:

- Función discriminante FSM (propuesta por FS Mikelberg $(13,14)$ y que incluye el CSM, la Variación de la altura de contorno y el Volumen de anillo neurorretiniano), y

- Función discriminante $R B$ (propuesta por $\mathrm{R}$ Bathija (15) y que incluye el CSM, la Variación de la altura de contorno, el Espesor de la capa de fibras nerviosas de la retina y el Área de anillo neurorretiniano).

\section{Test estadísticos}

Para la descripción de las características muestrales se emplearon la media y la desviación estándar.

El estudio estadístico de comparación de variables en la descripción de las características de cada uno de los grupos se llevó a cabo mediante análisis de la varianza entre grupos (ANOVA) para más de 2 grupos y test de Scheffé para ajuste de comparaciones múltiples. La comparación entre 2 grupos se llevó a cabo mediante el test $\mathrm{t}$ de Student.

El estudio estadístico de comparación de variables en el análisis topográfico papilar entre grupos de sujetos se llevó a cabo mediante análisis de la varianza entre grupos (ANOVA) y test de Scheffé para el ajuste de comparaciones múltiples.

Se fijó un valor de $\mathrm{p}<0,05$ como nivel de signifcación estadística que evidencia y contrasta diferencias entre grupos.

Se utilizó el programa SPSS 11.0 (SPSS for Windows, SPSS, Inc., Chicago, USA) para el tratamiento estadístico de los datos.

\section{RESULTADOS}

En la tabla I se recogen las características descriptivas de las poblaciones incluidas. Al comparar estas características entre los grupos, existieron diferencias estadísticamente significativas en los valores de PIO media, excavación papilar, y en los valores de los índices globales perimétricos (DM y CPSD).

Al comparar los diferentes parámetros en el grupo de sujetos glaucomatosos con los obtenidos en el grupo control se evidenciaron diferencias significativas en todas las variables papilares estudiadas $(\mathrm{p}<0,05)$ excepto en el área papilar, variación de la altura de contorno y variabilidad media (tabla II). En la comparación realizada entre el grupo de sujetos con glaucoma y el grupo de hipertensos oculares no se han evidenciado diferencias significativas en el disco papilar, profundidad media de la excavación, máxima profundidad de la excavación, variación de la altura de contorno, cociente excavación/papila horizontal, máxima depresión del contorno, altura de referencia $(p>0,05)$. Los restantes parámetros evaluados evidenciaron diferencias significativas $(\mathrm{p}<0,05)$ (tabla II).

Tabla I. Características del grupo control, hipertensión ocular y glaucoma, señalando los valores medios y las desviaciones estándar

\begin{tabular}{lcccccc}
\hline & \multicolumn{2}{c}{ Grupo Control $(\mathrm{n}=101)$} & \multicolumn{2}{c}{ Grupo HTO $(\mathrm{n}=247)$} & \multicolumn{2}{c}{ Grupo Glaucoma $(\mathrm{n}=102)$} \\
& \multicolumn{2}{c}{ Media } & $\mathrm{DE}$ & Media & $\mathrm{DE}$ & \multicolumn{2}{c}{ Media } & DE \\
\hline Edad & $55,53^{(3)}$ & 12,78 & $53,7^{(3)}$ & 12,39 & $62,9^{(1,2)}$ & 9,70 \\
A.V. & $0,857^{(2)}$ & 0,188 & 0,94 & 0,09 & 0,85 & 0,12 \\
PIO Basal & $14,6^{(2,3)}$ & 2,79 & $23,8^{(1)}$ & 3,21 & $24,2^{(1)}$ & 4,53 \\
Excavación & $0,87 / 5^{(2,3)}$ & $0,82 / 5$ & $2,55 / 5^{(1,3)}$ & $0,95 / 5$ & $3,46 / 5^{(1,2)}$ & $1,12 / 5$ \\
DM & $-0,56^{(3)}$ & 1,54 & $-0,38^{(3)}$ & 1,29 & $-6,81^{(1,2)}$ & 6,79 \\
CPSD & $1,03^{(3)}$ & 1,06 & $1,11^{(3)}$ & 0,90 & $4,95^{(1,2)}$ & 3,75 \\
\hline \hline
\end{tabular}

Diferencias estadísticamente significativas. (1) respecto al grupo Control; (2) respecto al grupo Hipertenso; (3) respecto al grupo Glaucoma. DM: defecto medio; CPSD: desviación estándar de patrón corregida. 
Tabla II. Parámetros estructurales (media y desviación estándar) obtenidos mediante HRT en sujetos normales, hipertensos oculares y glaucomas

\begin{tabular}{|c|c|c|c|c|c|c|c|c|c|}
\hline \multirow{2}{*}{ Parámetros HRT } & \multicolumn{3}{|c|}{ Normal $(n=101)$} & \multicolumn{3}{|c|}{ HTO $(n=247)$} & \multicolumn{3}{|c|}{ Glaucomas $(n=102)$} \\
\hline & Media & $\mathrm{DE}$ & $\mathrm{S}$ & Media & $\mathrm{DE}$ & $\mathrm{S}$ & Media & $\mathrm{DE}$ & $\mathrm{S}$ \\
\hline Área de disco & 2,23 & 0,47 & & 2,15 & 0,46 & & 2,1 & 0,46 & \\
\hline Área de excavacion & 0,52 & 0,4 & 2,3 & 0,75 & 0,39 & 1,3 & 0,96 & 0,49 & 1,2 \\
\hline Área de anillo & 1,71 & 0,39 & 2,3 & 1,4 & 0,3 & 1,3 & 1,14 & 0,43 & 1,2 \\
\hline Cociente de áreas excavación/disco & 0,22 & 0,14 & 2,3 & 0,34 & 0,14 & 1,3 & 0,45 & 0,2 & 1,2 \\
\hline Cociente de áreas anillo/disco & 0,78 & 0,14 & 2,3 & 0,66 & 0,14 & 1,3 & 0,55 & 0,2 & 1,2 \\
\hline Volumen de excavación & 0,13 & 0,15 & 2,3 & 0,22 & 0,16 & 1,3 & 0,29 & 0,25 & 1,2 \\
\hline Volumen de anillo & 0,48 & 0,21 & 2,3 & 0,37 & 0,16 & 1,3 & 0,27 & 0,15 & 1,2 \\
\hline Profundidad media de excavaión & 0,21 & 0,1 & 2,3 & 0,28 & 0,11 & 1 & 0,3 & 0,13 & 1 \\
\hline Profundidad máxima de excavación & 0,57 & 0,25 & 2,3 & 0,71 & 0,23 & 1 & 0,68 & 0,25 & 1 \\
\hline Variación de la altura del contorno & 0,41 & 0,11 & & 0,41 & 0,11 & & 0,39 & 0,13 & \\
\hline Índice de morfología de la excavación & $-0,19$ & 0,07 & 2,3 & $-0,15$ & 0,07 & 1,3 & $-0,09$ & 0,09 & 1,2 \\
\hline Espesor de la CFNR & 0,24 & 0,08 & 3 & 0,24 & 0,07 & 3 & 0,18 & 0,08 & 1,2 \\
\hline Área de sección de la CFNR & 1,27 & 0,43 & 3 & 1,27 & 0,41 & 3 & 0,92 & 0,44 & 1,2 \\
\hline Cociente excavación/papila horizontal & 0,45 & 0,24 & 2,3 & 0,57 & 0,18 & 1 & 0,62 & 0,22 & 1 \\
\hline Cociente excavación/papila vertical & 0,32 & 0,23 & 2,3 & 0,48 & 0,21 & 1,3 & 0,59 & 0,25 & 1,2 \\
\hline Máxima elevación del contorno & $-0,15$ & 0,1 & 2,3 & $-0,07$ & 0,11 & 1,3 & $-0,04$ & 0,16 & 1,2 \\
\hline Máxima depresión del contorno & 0,26 & 0,15 & 2,3 & 0,34 & 0,15 & 1 & 0,36 & 0,15 & 1 \\
\hline Línea de modulación de contorno temporal superior & 0,2 & 0,09 & 3 & 0,19 & 0,09 & 3 & 0,13 & 0,12 & 1,2 \\
\hline Línea de modulación de contorno temporal inferior & 0,19 & 0,1 & 3 & 0,19 & 0,09 & 3 & 0,11 & 0,11 & 1,2 \\
\hline Variabilidad media & 0,05 & 0,04 & 2 & 0,03 & 0,02 & 1,3 & 0,04 & 0,04 & 2 \\
\hline Altura de referencia & 0,28 & 0,15 & 2,3 & 0,37 & 0,15 & 1 & 0,33 & 0,14 & 1 \\
\hline Función FSM & 2,25 & 2,43 & 2,3 & 0,39 & 1,83 & 1,3 & $-1,03$ & 2,48 & 1,2 \\
\hline Función RB & 1,44 & 0,87 & 2,3 & 1,16 & 0,89 & 1,3 & 0,25 & 1,1 & 1,2 \\
\hline
\end{tabular}

Las columnas de la derecha muestran la significación de las diferencias del análisis de la varianza entre grupos (ANOVA). Se considera significativa una diferencia menor a $\mathrm{p}<0.05$. 1: significación con control; 2: significación con HTO; 3: significación con glaucoma.

Comparando el grupo de sujetos de hipertensos oculares con la población de sujetos normales se han encontrado diferencias significativas $(p<0,05)$ en la mayoría de las variables papilares exceptuando el disco papilar, la variación de la altura de contorno, el grosor medio de la capa de fibras, el área de corte de la capa de fibras nerviosas y en la líneas de modulación de contorno, temporal superior e inferior ( $\mathrm{p}>0,05)$ (tabla II).

Al comparar las mediciones de los diferentes parámetros estructurales papilares obtenidos en la población de sujetos hipertensos oculares sin evidencia de sospecha de daño papilar (hipertensos oculares) con respecto al grupo de sujetos controles, no se han evidenciado diferencias significativas en la mayoría de los mismos con excepción del área de disco, área de anillo, volumen de anillo, la elevación y depresión máxima de la línea de contorno, y la función discriminante FSM. Por el contrario se han encontrado diferencias significativas $(\mathrm{p}<0,05)$ en la casi totalidad de los parámetros estudiados (excepto área de disco, máxima profundidad de la excavación, variación de la altura del contorno y máxima depresión del contorno) al comparar los hipertensos oculares con los resultados observados en la población de sujetos glaucomatosos. Asimismo, la gran mayoría de los parámetros papilares (excepto área de anillo, volumen de anillo, variación de la altura de la línea de contorno, espesor medio de la CFNR, área de corte de la CFNR, máxima elevación y depresión del contorno) mostraron diferencias significativas al establecer la comparación con el grupo de sujetos con glaucoma pre-perimétrico (tabla III).

En relación al grupo de glaucomas pre-perimétricos los resultados del análisis han evidenciado una tendencia a la inversa, es decir al comparar los diferentes parámetros papilares con los obtenidos en el grupo control se han evidenciado diferencias significativas en todas las variables estudiadas $(\mathrm{p}<0,05)$ excepto en el área papilar, variación de la altura de contorno, espesor medio de la CFNR y área de corte de la CFNR. Sin embargo al establecer la comparación con respecto al grupo de sujetos con glaucoma en la gran mayoría de las variables papilares no se evidenciaron diferencias significativas con excepción del área de disco, área de anillo, volumen de anillo, espesor medio de la CFNR, área de corte 
Tabla III. Parámetros estructurales (media y desviación estándar) obtenidos mediante HRT en sujetos normales (grupo control), hipertensos oculares con papila normal, glaucomas pre-perimétricos y glaucomas

\begin{tabular}{|c|c|c|c|c|c|c|c|c|c|c|c|c|}
\hline \multirow[t]{2}{*}{ Parámetros HRT } & \multicolumn{3}{|c|}{$\begin{array}{l}\text { Normal } \\
(\mathrm{n}=101)\end{array}$} & \multicolumn{3}{|c|}{$\begin{array}{c}\text { HTO } \\
(n=126)\end{array}$} & \multicolumn{3}{|c|}{$\begin{array}{l}\text { Glaucoma preperimétrico } \\
\qquad(\mathrm{n}=121)\end{array}$} & \multicolumn{3}{|c|}{$\begin{array}{l}\text { Glaucomas } \\
(\mathrm{n}=102)\end{array}$} \\
\hline & Media & $\mathrm{DE}$ & S & Media & $\mathrm{DE}$ & S & Media & $\mathrm{DE}$ & S & Media & $\mathrm{DE}$ & S \\
\hline Área de disco & 2,23 & 0,47 & 2 & 1,96 & 0,42 & 1,3 & 2,36 & 0,42 & 2,4 & 2,1 & 0,46 & 3 \\
\hline Área de excavación & 0,52 & 0,4 & 3,4 & 0,53 & 0,32 & 3,4 & 0,98 & 0,32 & 1,2 & 0,96 & 0,49 & 1,2 \\
\hline Área de anillo & 1,71 & 0,39 & $2,3,4$ & 1,42 & 0,3 & 1,4 & 1,38 & 0,31 & 1,4 & 1,14 & 0,43 & $1,2,3$ \\
\hline Cociente de áreas excavación/disco & 0,22 & 0,14 & 3,4 & 0,26 & 0,13 & 3,4 & 0,41 & 0,11 & 1,2 & 0,45 & 0,2 & 1,2 \\
\hline Cociente de áreas anillo/disco & 0,78 & 0,14 & 3,4 & 0,74 & 0,13 & 3,4 & 0,59 & 0,11 & 1,2 & 0,55 & 0,2 & 1,2 \\
\hline Volumen de excavación & 0,13 & 0,15 & 3,4 & 0,14 & 0,13 & 3,4 & 0,29 & 0,16 & 1,2 & 0,29 & 0,25 & 1,2 \\
\hline Volumen de anillo & 0,48 & 0,21 & $2,3,4$ & 0,38 & 0,18 & 1,4 & 0,35 & 0,14 & 1,4 & 0,27 & 0,15 & $1,2,3$ \\
\hline Profundidad media d & 0,21 & 0,1 & 3,4 & 0,24 & 0,1 & 3,4 & 0,33 & 0,1 & 1,2 & 0,3 & 0,13 & 1,2 \\
\hline Profundidad máx. d & 0,57 & 0,25 & 3,4 & 0,66 & 0,24 & 3 & 0,76 & 0,21 & 1,2 & 0,68 & 0,25 & 1 \\
\hline Variación de la altura del contorno & 0,41 & 0,11 & & 0,42 & 0,11 & & 0,41 & 0,11 & & 0,39 & 0,13 & \\
\hline Índice de morfología de la excvación & $-0,19$ & 0,07 & 3,4 & $-0,18$ & 0,07 & 3,4 & $-0,11$ & 0,06 & 1,2 & $-0,09$ & 0,09 & 1,2 \\
\hline Espesor de la CFNR & 0,24 & 0,08 & 4 & 0,26 & 0,08 & 4 & 0,23 & 0,07 & 4 & 0,18 & 0,08 & $1,2,3$ \\
\hline Área de sección de la CFNR & 1,27 & 0,43 & 4 & 1,26 & 0,39 & 4 & 1,27 & 0,42 & 4 & 0,92 & 0,44 & $1,2,3$ \\
\hline Cociente excavación/papila horizontal & 0,45 & 0,24 & 3,4 & 0,49 & 0,19 & 3,4 & 0,65 & 0,14 & 1,2 & 0,62 & 0,22 & 1,2 \\
\hline Cociente excavación/papila vertical & 0,32 & 0,23 & 3,4 & 0,39 & 0,22 & 3,4 & 0,58 & 0,13 & 1,2 & 0,59 & 0,25 & 1,2 \\
\hline Máxima elevación del contorno & $-0,15$ & 0,1 & $2,3,4$ & $-0,09$ & 0,13 & 1,4 & $-0,05$ & 0,09 & 1 & $-0,04$ & 0,16 & 1,2 \\
\hline Máxima depresión del contorno & 0,26 & 0,15 & $2,3,4$ & 0,33 & 0,18 & 1 & 0,36 & 0,12 & 1 & 0,36 & 0,15 & 1 \\
\hline $\begin{array}{l}\text { Línea de modulación de contorno } \\
\text { Tem. Sup. }\end{array}$ & 0,2 & 0,09 & 4 & 0,2 & 0,09 & 4 & 0,19 & 0,09 & 4 & 0,13 & 0,12 & $1,2,3$ \\
\hline $\begin{array}{l}\text { Línea de modulación de contorno } \\
\text { Tem. Inf. }\end{array}$ & 0,19 & 0,1 & 4 & 0,21 & 0,09 & 4 & 0,18 & 0,09 & 4 & 0,11 & 0,11 & $1,2,3$ \\
\hline Variabilidad media & 0,05 & 0,04 & 2,3 & 0,03 & 0,02 & 1,4 & 0,03 & 0,02 & 1,4 & 0,04 & 0,04 & 2,3 \\
\hline Altura de referencia & 0,28 & 0,15 & 2,3 & 0,36 & 0,18 & 1 & 0,38 & 0,11 & 1 & 0,33 & 0,14 & \\
\hline Función FSM & 2,25 & 2,43 & $2,3,4$ & 0,98 & 1,88 & $1,3,4$ & $-0,22$ & 1,56 & $1,2,4$ & $-1,03$ & 2,48 & $1,2,3$ \\
\hline Función RB & 1,44 & 0,87 & 3,4 & 1,45 & 0,87 & 3,4 & 0,86 & 0,81 & $1,2,4$ & 0,25 & 1,1 & $1,2,3$ \\
\hline
\end{tabular}

Las columnas de la derecha muestran la significación de las diferencias del análisis de la varianza entre grupos (ANOVA). Se considera significativa una diferencia menor a p<0,05; 1: significación con control; 2: significación con HTO; 3: significación con glaucoma preperimétrico; 4: significación con glaucoma.

de la CFNR, función discriminante RB y función discriminante FSM (tabla III).

Al comparar los parámetros incluyendo los subgrupos de hipertensos según el resultado de la PALOC, no se apreciaron diferencias significativas en el área papilar entre ninguno de los 4 grupos de estudio. Tampoco se apreciaron diferencias en área de anillo neurorretiniano ni de excavación entre ambos grupos de hipertensión ocular, aunque el grupo de HTO con PALOC alterado presenta menor anillo neurorretiniano que el grupo de PALOC normal. Los volúmenes de anillo y excavación, así como las profundidades medias y máximas de la excavación muestran un comportamiento similar. Las diferencias entre los hipertensos oculares no son significativas entre ellos, pero sí con los grupos control y glaucoma. En el caso de la morfología de la excavación (CSM) sí se observan diferencias significativas entre ambos grupos de hipertensos. Los cocientes de excavación/papila muestran diferencias entre los subgrupos de HTO que no son significativas entre ellos, pero vuelven a mostrar significación con los grupos glaucoma y control al igual que ocurría con las áreas. En relación con las fórmulas multivariantes, el RB mostró diferencias significativas entre ambos grupos de HTO. La fórmula FSM escalona a los 4 grupos correctamente, sin mostrar diferencias significativas entre los subgrupos de HTO.

\section{DISCUSIÓN}

La primera aproximación al análisis diferencial de las características papilares se centra sobre las áreas de anillo neurorretiniano y de excavación de los sujetos estudiados. Ambos grupos de parámetros muestran diferencias estadísticamente significativas entre los sujetos normales, hipertensos oculares y glaucomatosos, disminuyendo sus áreas de anillo progresivamente desde el grupo normal al de 
Tabla IV. Parámetros estructurales (media y desviación estandar) obtenidos mediante HRT en sujetos normales, hipertensos oculares con PALOC normal, hipertensos oculares con PALOC alterada y glaucomas

\begin{tabular}{|c|c|c|c|c|c|c|c|c|c|c|c|c|}
\hline \multirow[t]{2}{*}{ Parámetros HRT } & \multicolumn{3}{|c|}{$\begin{array}{l}\text { Normal } \\
(\mathrm{n}=101)\end{array}$} & \multicolumn{3}{|c|}{$\begin{array}{l}\text { HTO + PALOC } \\
\text { normal }(n=126)\end{array}$} & \multicolumn{3}{|c|}{$\begin{array}{c}\text { HTO + PALOC alterado } \\
(\mathrm{n}=46)\end{array}$} & \multicolumn{3}{|c|}{$\begin{array}{c}\text { Glaucomas } \\
(\mathrm{n}=102)\end{array}$} \\
\hline & Media & $\mathrm{DE}$ & S & Media & $\mathrm{DE}$ & S & Media & $\mathrm{DE}$ & $S$ & Media & $\mathrm{DE}$ & $\mathrm{S}$ \\
\hline Área de disco & 2,23 & 0,47 & & 2,14 & 0,49 & & 2,22 & 0,43 & & 2,1 & 0,46 & \\
\hline Área de excavación & 0,52 & 0,4 & $2,3,4$ & 0,73 & 0,4 & 1,4 & 0,89 & 0,33 & 1 & 0,96 & 0,49 & 1,2 \\
\hline Área de anillo & 1,71 & 0,39 & $2,3,4$ & 1,41 & 0,32 & 1,4 & 1,33 & 0,25 & 1,4 & 1,14 & 0,43 & $1,2,3$ \\
\hline Cociente de áreas excavación/disco & 0,22 & 0,14 & $2,3,4$ & 0,33 & 0,14 & 1,4 & 0,39 & 0,11 & 1 & 0,45 & 0,2 & 1,2 \\
\hline Cociente de áreas anillo/disco & 0,78 & 0,14 & $2,3,4$ & 0,67 & 0,14 & 1,4 & 0,61 & 0,11 & 1 & 0,55 & 0,2 & 1,2 \\
\hline Volumen de excavación & 0,13 & 0,15 & $2,3,4$ & 0,2 & 0,16 & 1,4 & 0,25 & 0,13 & 1 & 0,29 & 0,25 & 1,2 \\
\hline Volumen de anillo & 0,48 & 0,21 & $2,3,4$ & 0,38 & 0,16 & 1,4 & 0,31 & 0,12 & 1 & 0,27 & 0,15 & 1,2 \\
\hline Profundidad media de excavación & 0,21 & 0,1 & $2,3,4$ & 0,28 & 0,1 & 1 & 0,3 & 0,09 & 1 & 0,3 & 0,13 & 1 \\
\hline Profundidad máx. de excavación & 0,57 & 0,25 & $2,3,4$ & 0,71 & 0,21 & 1 & 0,73 & 0,19 & 1 & 0,68 & 0,25 & 1 \\
\hline Variación de la altura de contorno & 0,41 & 0,11 & & 0,41 & 0,09 & & 0,39 & 0,11 & & 0,39 & 0,13 & \\
\hline Índice de morfología de la excavación & $-0,19$ & 0,07 & $2,3,4$ & $-0,15$ & 0,07 & $1,3,4$ & $-0,12$ & 0,08 & 1,2 & $-0,09$ & 0,09 & 1,2 \\
\hline Espesor de la CFNR & 0,24 & 0,08 & 4 & 0,25 & 0,06 & 4 & 0,21 & 0,07 & & 0,18 & 0,08 & 1,2 \\
\hline Área de sección de la CFNR & 1,27 & 0,43 & 4 & 1,29 & 0,39 & 4 & 1,12 & 0,38 & & 0,92 & 0,44 & 1,2 \\
\hline Cociente horizontal excavación/disco & 0,45 & 0,24 & $2,3,4$ & 0,55 & 0,17 & 1 & 0,64 & 0,14 & 1 & 0,62 & 0,22 & 1 \\
\hline Cociente vertical excavación/disco & 0,32 & 0,23 & $2,3,4$ & 0,48 & 0,2 & 1,4 & 0,57 & 0,15 & 1 & 0,59 & 0,25 & 1,2 \\
\hline Máxima elevación del contorno & $-0,15$ & 0,1 & $2,3,4$ & $-0,07$ & 0,09 & 1 & $-0,06$ & 0,11 & 1 & $-0,04$ & 0,16 & 1 \\
\hline Máxima depresión del contorno & 0,26 & 0,15 & 2,4 & 0,34 & 0,12 & 1 & 0,33 & 0,13 & & 0,36 & 0,15 & 1 \\
\hline $\begin{array}{l}\text { Línea de modulación de contorno } \\
\text { Tem. Sup. }\end{array}$ & 0,2 & 0,09 & 4 & 0,19 & 0,08 & 4 & 0,16 & 0,09 & & 0,13 & 0,12 & 1,2 \\
\hline $\begin{array}{l}\text { Línea de modulación de contorno 0,19 } \\
\text { Tem. Inf. }\end{array}$ & 0,1 & 4 & 0,2 & 0,09 & 4 & 0,16 & 0,07 & 4 & 0,11 & 0,11 & $1,2,3$ & \\
\hline Variabilidad media & 0,05 & 0,04 & 2,3 & 0,03 & 0,02 & 1 & 0,03 & 0,03 & 1 & 0,04 & 0,04 & \\
\hline Altura de referencia & 0,28 & 0,15 & 2 & 0,37 & 0,11 & 1 & 0,34 & 0,13 & & 0,33 & 0,14 & \\
\hline Función FSM & 2,25 & 2,43 & $2,3,4$ & 0,57 & 1,81 & 1,4 & $-0,41$ & 1,52 & 1 & $-1,03$ & 2,48 & 1,2 \\
\hline Función RB & 1,44 & 0,87 & 3,4 & 1,24 & 0,81 & 3,4 & 0,74 & 0,81 & $1,2,4$ & 0,25 & 1,1 & $1,2,3$ \\
\hline
\end{tabular}

Las columnas de la derecha muestran la significación de las diferencias del ANOVA. Se considera significativa una p<0,05; 1: significación con control; 2: significación con HTO + PALOC normal; 3: significación con HTO + PALOC alterado; 4: significación con glaucoma.

glaucomas y aumentando sus áreas de excavación. La diferencia clasificatoria entre el grupo hipertenso ocular y el glaucoma es la presencia de defectos en la perimetría convencional, por tanto, el comportamiento de estos parámetros, en presencia de áreas papilares sin diferencias significativas, indican que existe pérdida de anillo neurorretiniano en sujetos hipertensos oculares antes de que puedan ser detectadas pérdidas funcionales en la perimetría convencional. Estos resultados enfatizan la necesidad de seguir buscando técnicas que puedan detectar pérdidas en fases más iniciales de la enfermedad.

El estudio de las proporciones papilares mostró las mismas diferencias significativas que los estudios de áreas de anillo y excavación. Entre estos cocientes, el cociente del área y de la excavación/papila verticales parecen ser superiores a los horizontales.

En relación con las mediciones que involucran el cálculo de profundidades, espesores y volúmenes papilares, los resultados están en concordancia con los obtenidos con la mayoría de autores, como
Uchida y col (16). Estos parámetros ven condicionados sus resultados a la definición y posición del plano de referencia.

Respecto al índice de morfología de la excavación, CSM, ha mostrado ser un parámetro con gran capacidad discriminante del daño, mostrando diferencias significativas entre todos los grupos de estudio. Estos resultados están en concordancia con la mayoría de autores (13-20) quienes consideran este parámetro como uno de los que mejor discriminan entre sujetos normales, hipertensos oculares y glaucomas. Por ejemplo, observamos que Uchida y col (16) considera que los parámetros con mayor valor diagnóstico en su estudio fueron el CSM y el cociente excavación/papila.

Los parámetros de espesor de la capa de fibras nerviosas de la retina y el área de corte de la misma no muestran diferencias significativas entre el grupo normal y el de hipertensos oculares, mientras que sí se observan diferencias significativas entre hipertensos y glaucomas. Posiblemente, estos parámetros no ofrecen gran utilidad en el diagnóstico 
precoz de la enfermedad (16) aparte de estar influenciados por la posición del plano de referencia y no por el espesor real de la capa de fibras.

El programa empleado para el estudio incluye 2 funciones discriminantes de daño glaucomatoso (FSM y RB) que son el resultado de la ponderación diagnóstica de algunas de las variables más rentables según los autores que las propusieron. En nuestro estudio dichas funciones multivariantes permitieron discriminar la presencia de daño glaucomatoso. La función FSM se comportó mejor que la función RB para la diferenciación de las subpoblaciones de sujetos con menores niveles de daño glaucomatoso.

El tamaño de la papila es uno de los parámetros de mayor influencia en la interpretación de los demás resultados. La gran variabilidad interindividual que pueden presentar los discos ópticos es una de las fuentes de error más importantes en el diagnóstico del glaucoma. El análisis general de los grupos normal, hipertenso ocular y glaucoma no mostró diferencias estadísticamente significativas entre las 3 muestras de sujetos estudiados, lo que facilita la interpretación de los demás parámetros y potencia el valor diagnóstico de las diferencias encontradas.

En este trabajo hemos pretendido estudiar la rentabilidad de la evaluación subjetiva papilar y de la perimetría de longitud de onda corta en la detección de sujetos hipertensos oculares con mayor riesgo de desarrollar la enfermedad y que por tanto más se pueden beneficiar de un inicio del tratamiento.

La introducción de los criterios de evaluación subjetiva papilar ha mostrado diferencias estadísticamente significativas entre ambos subgrupos en área papilar.

Estas diferencias se trasladan al área de excavación. Asimismo, la influencia del tamaño de disco resta importancia a las diferencias halladas en los cocientes excavación/papila, que también muestran diferencias significativas entre ambos grupos. Los volúmenes de anillo y de excavación se comportan de una manera parecida a las áreas. Sin embargo, el área de anillo, muestra diferencias no significativas entre ambos subgrupos.

El hecho de que el índice de morfología de la excavación muestre diferencias significativas entre ambos grupos apoya la idea de que existe mayor daño estructural en nuestro grupo de glaucoma preperimétrico que en el grupo de hipertensos. De hecho, el CSM es uno de los parámetros más independientes del tamaño de disco y del plano de referencia. El grupo de hipertensos no mostró diferencias significativas con el grupo normal, de la misma manera que el grupo de glaucoma preperimétrico no tuvo diferencias significativas con el grupo glaucoma, mientras que entre ellos las diferencias sí fueron significativas. La casi perfecta segregación de los sujetos de ambos grupos refuerza el valor del diagnóstico a partir de la evaluación subjetiva del nervio óptico, dado el gran valor que ha demostrado el CSM en múltiples estudios anteriores (13-20). Por otra parte, el hecho de que no existan diferencias entre glaucoma preperimétrico y glaucoma sugiere que el CSM se ve alterado en fases iniciales de la enfermedad, por tanto, este parámetro puede tener especial importancia en el diagnóstico precoz de la misma.

Las funciones discriminantes han demostrado una adecuada capacidad discriminatoria del daño glaucomatoso. Evidentemente, el comportamiento de estas fórmulas basa gran parte de su éxito en que ambas incluyen el CSM.

Al clasificar los hipertensos oculares según su comportamiento funcional en la PALOC no se han observado diferencias estadísticamente significativas en el área papilar entre ninguno de los grupos de estudio. Esto pone de manifiesto el sesgo que introduce la influencia de este parámetro en la evaluación subjetiva del nervio óptico, siendo más fácil diagnosticar la enfermedad desde el punto de vista estructural en una papila grande que en una papila pequeña.

El índice de morfología de la excavación muestra diferencias significativas incluso entre ambos grupos de hipertensos (con o sin alteración en la PALOC) y subdivide correctamente los 4 grupos de este apartado, mostrando una CSM menos negativa cuanto más alejado de la normalidad está el grupo.

Sólo la función RB muestra diferencias significativas entre ambos grupos de hipertensión ocular, por lo que esta función podría ser más sensible a la pérdida funcional glaucomatosa detectada por la PALOC.

El empleo del topógrafo papilar HRT proporciona una moderada capacidad de discriminación entre sujetos normales, hipertensos oculares y glaucomatosos. El empleo de herramientas diagnósticas para la detección precoz del daño glaucomatoso (como la perimetría de longitud de onda corta) mejora la capacidad discriminatoria del HRT.

\section{BIBLIOGRAFÍA}

1. Jonas JB, Fernandez MC, Sturmer J. Pattern of glaucomatous neuroretinal rim loss. Ophthalmology 1993; 100: 63-68. 
2. Jonas JB, Gusek GC, Naumann GO. Optic disc, cup and neuroretinal rim size, configuration and correlations in normal eyes. Invest Ophthalmol Vis Sci 1988; 29: 1151-1158.

3. Polo V, Abecia E, Pablo LE, Pinilla I, Larrosa JM, Honrubia FM. Short-wavelength automated perimetry and retinal nerve fiber layer evaluation in suspects cases of glaucoma. Arch Ophthalmol 1998; 116: 1295-1298.

4. Sommer A, Katz J, Quigley HA, Miller NR, Robin AL, Richter RC,et al. Clinically detectable nerve fiber layer atrophy predeces the onset of glaucomatous field loss. Arch Ophthalmol 1991; 109: 77-83.

5. Heijl A, Lindgren G, Olsson J, Asman P. Visual field interpretation with empiric probability maps. Arch Ophthalmol 1989; 107: 204-208.

6. Polo V, Larrosa JM, Pinilla I, Perez S, Gonzalvo F, Honrubia FM. Predictive value of short-wavelength automated perimetry: a 3-year follow-up study. Ophthalmology 2002; 109: 761-765.

7. Balazsi AG, Drance SM, Schulzer M Douglas GR. Neuroretinal rim area in suspected glaucoma and early chronic open-angle glaucoma. Correlation with parameters of visual function. Arch Ophthalmol 1984; 102: 1011-1014.

8. Airaksinen PJ, Drance SM, Schulzer M. Neuroretinal rim area in early glaucoma. Am J Ophthalmol 1985; 99: 1-4.

9. Abecia E, Honrubia FM. Retinal nerve fiber layer defects and automated perimetry evaluation in ocular hypertensives. Int Ophthalmol 1992; 16: 239-242.

10. Tuulonen A, Airaksinen PJ. Initial glaucomatous optic disk and retinal nerve fiber layer abnormalities and their progression. Am J Ophthalmol 1991; 111: 485-490.

11. Airaksinen PJ, Tuulonen A, Alanko HI. Rate and pattern of neuroretinal rim area decrease in ocular hypertension and glaucoma. Arch Ophthalmol 1992; 110: 206-210.
12. Jonas JB, Konigsreuther KA. Optic disk appearance in ocular hypertensive eyes. Am J Ophthalmol 1994; 117: 732-740.

13. Mikelberg FS, Parfitt CM, Swindale NV, Grahan SL, Drance SM, Gosine R. Ability of the heidelberg retina tomograph to detect early glaucomatous visual field loss. J Glaucoma 1995; 4: 242-247.

14. Iester M, Mikelberg FS, Drance SM. The effect of optic disc size on diagnostic precision with the Heidelberg retina tomograph. Ophthalmology 1997; 104: 545-548.

15. Bathija R, Zangwill L, Berry CC, Sample PA, Weinreb RN. Detection of early glaucomatous structural damage with confocal scanning laser tomography. J Glaucoma 1998; 7: 121-127.

16. Uchida H, Brigatti L, Caprioli J. Detection of structural damage from glaucoma with confocal laser image analysis. Invest Ophthalmol Vis Sci 1996; 37: 2393-2401.

17. Kamal DS, Viswanathan AC, Garway-Heath DF, Hitchings RA, Poinoosawmy D, Bunce C. Detection of optic disc change with the Heidelberg retina tomograph before confirmed visual field change in ocular hypertensives converting to early glaucoma. Br J Ophthalmol 1999; 83: 290-294.

18. Wollstein G, Garway-Heath DF, Hitchings RA. Identification of early glaucoma cases with the scanning laser ophthalmoscope. Ophthalmology 1998; 105: 1557-1563.

19. Brigatti L, Caprioli J. Correlation of visual field with scanning confocal laser optic disc measurements in glaucoma. Arch Ophthalmol 1995; 113: 1191-1194.

20. Tsai CS, Zangwill L, Sample PA, Garden V, Bartsch D, Weinreb RN. Correlation of peripapillary retinal height and visual field in glaucoma and normal subjects. J Glaucoma 1995; 4: 110-116. 